\title{
Experiencias y conclusiones en el desarrollo y validación de instrumentos de evaluación enfocados a la adquisición de la competencia transversal 'CT13 Instrumental Específica' en asignaturas de Ingeniería Mecánica
}

\author{
A.M. Pedrosaa, , A. Besa ${ }^{\text {a,2, }}$ M.J. Rupérez ${ }^{a, 3}$, E.M. Sánchez-Orgaz ${ }^{a, 4}$ J. Giner ${ }^{a, 5}$, S. Martínez- \\ Sanchís $^{\mathrm{a}, 6}$, M.P. Vila ${ }^{\mathrm{a}, 7}$, A. Rovira ${ }^{\mathrm{a}, 8}$, J.J. Ródenasa ${ }^{\mathrm{a}, 9}$, E. Nadal ${ }^{\mathrm{a}, 10}$, J. Martínez-Casas ${ }^{\mathrm{a}, 11}$, A. Pruna ${ }^{\mathrm{a}, 12}$, \\ A. Sonseca ${ }^{\mathrm{a}, 13}$, E. Lozano-Mínguez ${ }^{\mathrm{a}, 14}$, O. Sahuquillo ${ }^{\mathrm{a}, 15}$, R. Benavente ${ }^{\mathrm{a}, 16}$ y V.T. Andrés ${ }^{\mathrm{a}, 17}$ \\ a'Departamento de Ingeniería Mecánica y de Materiales. Universitat Politècnica de València, ${ }^{1}$ anpedsan@dimm.upv.es, \\ ${ }^{2}$ abesa@mcm.upv.es, ${ }^{3}$ mjrupere@upvnet.upv.es, ${ }^{4}$ evsncor@upvnet.upv.es, ${ }^{5}$ juanginer@upv.es, ${ }^{6}$ sanmars1@upv.es, \\ 7mavitor2@upvnet.upv.es, 8 arovira@mcm.upv.es, 9jjrodena@mcm.upv.es, ${ }^{10}$ ennaso@upvnet.upv.es, \\ 11 jomarc12@mcm.upv.es,_12apruna@itm.upv.es,_13agsonol@upvnet.upv.es, ${ }^{14}$ eslomin@upv.es, \\ 15 ossana@upvnet.upv.es, ${ }^{16}$ rutbmr@upvnet.upv.es, ${ }^{17}$ vicanrui@etsid.upv.es
}

\section{Abstract}

This paper presents the results of the experience carried out in the lab-sessions of several subjects of Mechanical Engineering. The aim of this work is to find a common methodology focused on the acquisition and evaluation of the Specific Instrumental Transversal Competence (CT13 in the UPV institutional project).

The work has been applied in two types of lab-sessions: computer lab and laboratory. The instrumentation used in each of them has a different nature, while the former is focused on the use and comprehension of a numerical calculation software, in the latter the students must understand the management and purpose of some experimental measurement equipments.

In the computer sessions, after an accompanied learning, the students must carry out and justify the steps followed in solving a numerical problem. The evaluation takes into account the level of adequacy of the steps followed throughout the process. In the laboratory practices, the students must demonstrate the ability to correctly select the proper equipment based on the parameter to be measured as well as to define the sequence of treatments in the measurement process. The measurement of the level of acquisition of the CT13 is done through an individual questionnaire.

Keywords: methodology, evaluation, transversal competence, specific instrumental, ANSYS

\section{Resumen}

En este trabajo se presentan los resultados de la experiencia llevada a cabo en las prácticas de diversas asignaturas de Ingeniería Mecánica. El objetivo final es encontrar una metodología unificada enfocada a la adquisición y evaluación de la Competencia Transversal Instrumental Especifica (CT13 en el proyecto institucional de la UPV).

Se ha trabajado con dos tipos de prácticas: informáticas y de laboratorio. La instrumentación empleada en cada una de ellas tiene distinta naturaleza, mientras las primeras están enfocadas al manejo de un programa de cálculo numérico, en las segundas los estudiantes deben comprender el manejo y la finalidad de diversos equipos de medida experimental.

En las prácticas informáticas los alumnos, tras un aprendizaje acompañado, deben ser capaces de realizar y justificar los pasos seguidos en la resolución de un problema numérico. En la evaluación se tiene en cuenta el nivel de adecuación de los pasos seguidos a lo largo del proceso. En las prácticas de laboratorio los alumnos deben demostrar la capacidad de seleccionar correctamente 
Experiencias y resultados en el desarrollo y validación de instrumentos de evaluación enfocados a la adquisición de la competencia transversal 'CT13 Instrumental Especifica' en asignaturas de Ingeniería Mecánica

el equipo adecuado en función del parámetro a medir, así como definir la secuencia de tratamientos en el proceso experimental. La medida del nivel de adquisición de la CT13 se realiza con un cuestionario individual.

Palabras clave: metodología, evaluación, competencia transversal, instrumental especifica, ANSYS

\section{Introducción}

Hoy en día es indiscutible la importancia del desarrollo de las competencias transversales de carácter genérico en todos los niveles de educación. Dichas competencias consisten, entre otras, en habilidades técnicas, profesionales o interpersonales, y son muy tenidas en cuenta en el ámbito profesional, siendo cada vez más críticas para la inserción de los estudiantes en el mundo laboral (Andrews, 2008; Entwistle, 2004 ; Kelly, 2001; Rieckmann, 2012). Por esta razón, el trabajo y la evaluación de las competencias transversales están incluidos en la mayoría de los marcos de educación superior del mundo (Murias, 2007; Sursock, 2010; Young, 2010). En Europa se han incorporado siguiendo las líneas marcadas por el proyecto Tuning (Beneitone, 2007; Tunning Project) nacido al amparo del Espacio Europeo de Educación Superior (EEES). En este escenario, la Universitat Politècnica de València (UPV), lleva años trabajando en su propio proyecto institucional "Competencias Transversales" (UPV) orientado a complementar la formación específica de cada titulación en capacidades o habilidades demandadas en los perfiles profesionales actuales. Dicho proyecto abarca un ámbito más amplio que el puramente académico integrando aspectos sociales, éticos, de comunicación, trabajo en equipo, etc. Las acciones llevadas a cabo en el contexto del proyecto institucional tienen como objetivo principal la búsqueda de la excelencia de los egresados, tanto de cara a los empleadores como a las acreditaciones nacionales (ANECA) e internacionales (ABET).

Las iniciativas presentadas en este trabajo surgen en el marco del programa Aprendizaje y Docencia (A+D) impulsado por los vicerrectorados de Recursos Digitales y Documentación (VRED) y Estudios, Calidad y Acreditación (VECA) de la UPV. A raíz de la convocatoria de Proyectos de Innovación y Mejora Educativa (PIME), el profesorado de diversas asignaturas del Departamento de Ingeniería Mecánica y de Materiales (DIMM) de la misma universidad ha puesto en marcha una serie de actividades enfocadas a la mejora de la adquisición de la competencia transversal CT13 “Instrumental Específica” (UPV). Dicha competencia está orientada al uso de herramientas y tecnologías necesarias para la práctica profesional. El objetivo principal del trabajo llevado a cabo es la búsqueda de una metodología común tanto en las actividades para la adquisición de las destrezas como en su evaluación.

La experiencia se ha llevado a cabo en diversas asignaturas del DIMM. Tales asignaturas pueden clasificarse en dos grupos según el tipo de prácticas. Por un lado, las asignaturas del área de ingeniería mecánica realizan prácticas informáticas enfocadas al aprendizaje del manejo del programa ANSYS, el cual permite realizar el modelo numérico de un problema mecánico mediante el Método de Elementos Finitos (MEF) (Zienkiewicz, 2006). Por otro lado, en las asignaturas del área de ciencia de materiales e ingeniería metalúrgica las prácticas son de laboratorio y en ellas los alumnos deben conocer y aprender a manejar equipos de medida para determinar algunas propiedades mecánicas de los materiales (dureza, acritud, etc.). En todos los casos, la instrumentación sobre la que se trabaja se utiliza habitualmente en el desempeño profesional de la ingeniería mecánica. 


\section{Objetivos}

Los objetivos del presente trabajo se centran en la búsqueda y desarrollo de actividades que permitan evaluar la competencia transversal CT13 de forma similar en las prácticas de distintas asignaturas pertenecientes al DIMM. En este trabajo, esta competencia se ha enfocado al uso de herramientas y tecnologías necesarias para la práctica profesional asociada a la ingeniería mecánica.

Se ha trabajado en asignaturas con prácticas informáticas con el programa de elementos finitos ANSYS y en asignaturas con prácticas de laboratorio en las que se maneja instrumentación de caracterización experimental mecánica de materiales.

Por tanto, el objetivo final de este trabajo es formar al alumno en su capacidad de identificar las herramientas más adecuadas, conocer sus utilidades y poder integrarlas y combinarlas para resolver problemas, llevar a cabo proyectos o experimentos.

En la Sección 3 se detalla el desarrollo de las actividades y la metodología seguida para cada tipo de prácticas. En la Sección 4 se mostrarán los resultados más relevantes y, finalmente, en la Sección 5 las conclusiones.

\section{Desarrollo de la innovación}

Al tratarse de una competencia centrada en el uso de herramientas orientadas en la práctica profesional, el trabajo desarrollado se ha llevado a cabo exclusivamente en las sesiones de prácticas de las asignaturas seleccionadas. La propuesta desarrollada se ha elaborado conjuntamente por los participantes en las prácticas tanto informáticas como de laboratorio y presentan elementos en común. En aras de una mayor claridad, se expone por separado la experiencia de los dos tipos de prácticas.

\subsection{Prácticas informáticas}

\subsubsection{Descripción del programa informático}

En el diseño de componentes de máquinas es necesario, por un lado, la selección de un material adecuado, por otro, la realización del modelo de las condiciones de contorno y, por último, la aplicación de criterios de fallo adecuados. Estos aspectos son abordados en las sesiones teóricas de las asignaturas. Los cálculos asociados se pueden llevar a cabo con programas informáticos dotados de módulos de cálculo mediante elementos finitos.

Partiendo de la base de los conocimientos teóricos abordados en el aula, en las sesiones prácticas de las asignaturas se introduce el uso de uno de los programas comerciales de elementos finitos más completos que existen en el mercado: ANSYS. De esta forma, el enfoque de las sesiones prácticas tiene sentido doble: por un lado, se ofrecen como complemento a los conceptos teóricos, a la vez que cada una de las instrucciones contenidas en los cuadernillos está debidamente justificada bajo un punto de vista teórico ofreciendo, siempre que sea posible, diferentes alternativas valorando la adecuación de cada una de ellas.

\subsubsection{Asignaturas}

Entre las asignaturas se encuentran algunas que tienen como objetivo dar a conocer el propio MEF o aquellas pertenecientes al ámbito del Diseño de Máquinas (DM) en las que se emplea el MEF como herramienta de trabajo. En la Tabla 1 se proporcionan detalles de todas ellas. 
Experiencias y resultados en el desarrollo y validación de instrumentos de evaluación enfocados a la adquisición de la competencia transversal 'CT13 Instrumental Especifica' en asignaturas de Ingeniería Mecánica

Tabla 1. Asignaturas con prácticas informáticas

\begin{tabular}{|c|c|c|c|c|c|}
\hline $\begin{array}{c}\text { Asignatura, Curso- } \\
\text { Cuatrimestre, Titulación }{ }^{1}\end{array}$ & Ámbito & $\begin{array}{l}\text { Alumnos por } \\
\text { grupo de } \\
\text { prácticas } \\
\text { (promedio) }\end{array}$ & $\begin{array}{l}\quad \mathrm{N}^{\circ} \text { de } \\
\text { alumnos en } \\
\text { asignatura }\end{array}$ & $\begin{array}{l}\mathrm{N}^{0} \text { de } \\
\text { sesiones de } \\
\text { prácticas } \\
\text { (ANSYS) }\end{array}$ & $\begin{array}{l}\text { Duración } \\
\text { de la } \\
\text { sesión (h) }\end{array}$ \\
\hline $\begin{array}{c}\text { Métodos Numéricos }(\mathrm{MN}), 3^{\circ} \mathrm{A} \text {, } \\
\text { GIB }\end{array}$ & MEF & 20 & 60 & 2 & 2.5 \\
\hline $\begin{array}{c}\text { Técnicas computacionales en } \\
\text { Ingeniería Mecánica (TCIM), } \\
4^{\circ} \mathrm{A}, \mathrm{GIM}\end{array}$ & MEF & 27 & 54 & 15 & 2 \\
\hline $\begin{array}{l}\text { Vibraciones Mecánicas (VM), } \\
4^{\circ} \mathrm{A}, \mathrm{GIM}\end{array}$ & $\mathrm{DM}$ & 23 & 165 & 1 & 2 \\
\hline $\begin{array}{c}\text { Tecnología de Máquinas (TM), } \\
3^{\circ} \mathrm{A} \text {, GITI }\end{array}$ & DM & 18 & 280 & 2 & 3 \\
\hline $\begin{array}{l}\text { Tecnología de Máquinas para } \\
\text { Nivelación (TMN), } 1^{\circ} \mathrm{A}, \text { MUII }\end{array}$ & $\mathrm{DM}$ & 14 & 42 & 3 & 2.5 \\
\hline $\begin{array}{l}\text { Comportamiento de Materiales } \\
\text { en Servicio (CMS), } 1^{\circ} \mathrm{A} \text {, MUIM }\end{array}$ & $\mathrm{DM}$ & 19 & 19 & 3 & 3 \\
\hline
\end{tabular}

\subsubsection{Metodología}

A pesar de pertenecer a diferentes titulaciones y asignaturas, todas las sesiones de prácticas involucradas en este trabajo tienen una estructura común. En todas ellas se plantea un problema que es distinto en cada asignatura/sesión ya que se adapta a los contenidos y nivel correspondientes. Los problemas abordados tienen en común que su planteamiento se concreta en una determinada ecuación en derivadas parciales (EDP) que, junto con el conjunto de condiciones de contorno, conducen a una solución numérica particular. La estructura de las sesiones prácticas hasta la fecha consiste en proporcionar al alumno un guion muy detallado en el que se explican los pasos a seguir durante el desarrollo de la sesión para la obtención del resultado numérico que muchas veces pueden comparar con resultados bibliográficos. En el cuadernillo de prácticas, se explica tanto el funcionamiento del programa como la justificación de los resultados parciales que se van obteniendo con el objetivo de que el alumno se familiarice con problemas presentes en el campo de la ingeniería mecánica.

Sin embargo, al plantear las sesiones prácticas siguiendo esta estructura, la evaluación de la CT13 resulta deficiente, al no mostrar si el alumno ha adquirido la autonomía suficiente para poder resolver problemas análogos por su cuenta. Por este motivo, en el primer curso de trabajo se decidió plantear una actividad alternativa al final de cada sesión de prácticas. La incorporación de este ejercicio sin pautar permitiría ver la evolución del alumno frente a un problema distinto.

La actividad está basada en la experiencia de la asignatura TCIM en la que se profundiza en las técnicas de cálculo numérico en las sesiones de teoría. Esta asignatura tiene más sesiones de prácticas que el resto de las involucradas en este estudio (ver Tabla 1) y hacen el ejercicio no guiado en la última sesión.

\footnotetext{
${ }^{1}$ GIB: Grado en Ingeniería Biomédica; GITI: Grado en Ingeniería en Tecnologías Industriales; MUII: Máster Universitario en Ingeniería Industrial; MUIM: Máster Universitario en Ingeniería Mecatrónica; GIM Grado en Ingeniería Mecánica
} 
Evidentemente, se ha tenido en cuenta que el nivel de exigencia no podía ser el mismo en las asignaturas del ámbito de DM. La asignatura TCIM forma parte de la mención de diseño de máquinas del GIM, por lo que tiene carácter optativo. El aprendizaje en el manejo del programa ANSYS y en el conocimiento de la teoría del MEF se ha aprovechado en la asignatura VM, que tiene carácter obligatorio. En la experiencia descrita en (Nadal, 2020) se comprobó como las capacidades en el manejo de ANSYS de los alumnos que habían cursado TCIM se difundió entre el resto de los alumnos de la promoción y sirvió para que todos resolvieran un problema avanzado que incluía un cálculo dinámico de un sistema mecánico.

Además, esta primera experiencia se hizo a pequeña escala en las asignaturas MN, TMN y CMS con pocos alumnos con el fin de comprobar la eficacia. El problema a resolver está basado en la resolución de la misma EDP, pero aplicada a una geometría distinta incluso, en ocasiones, con condiciones de contorno distintas.

El principal objetivo que se busca con la incorporación de esta nueva actividad no guiada es que la actitud del alumno durante las sesiones de prácticas sea más participativa y proactiva, la estrategia es ayudar a que se despierte el interés del estudiante en aprender qué implica cada uno de los pasos y decisiones que debe tomar en el transcurso del trabajo en lugar de limitarse a reproducirlos en su ordenador. Este aprendizaje es fundamental para resolver correctamente el problema individual que se les propone ya que implica seguir correctamente los pasos correspondientes dentro del programa ANSYS, tales son entre otros: configurar los diversos menús del programa, definir el dominio aplicando simplificaciones, elegir adecuadamente el tipo y tamaño de elementos con los que se discretiza el dominio, definir las características de los materiales, aplicar condiciones de contorno, etc. De alguna manera se pretende hacer consciente al alumno de su propia responsabilidad respecto al aprendizaje propuesto en las sesiones de prácticas. Además, la incorporación de esta novedad ha tenido un coste pequeño porque se ha podido mantener la estructura de gran parte de la sesión de prácticas y de los recursos docentes. El trabajo de adaptación ha supuesto revisar los cuadernillos de prácticas, modificando aquellos párrafos más difíciles de entender e incorporando vídeos explicativos con la ejecución de una secuencia de comandos para facilitar su localización en la pantalla. Por último, se ha elaborado la batería de ejercicios y las tareas de entrega con apartados en los que se pide el resultado numérico del problema propuesto, ficheros del programa y la justificación del proceso seguido. Los plazos de entrega han tenido que ser flexibles, en la etapa pre-COVID ante posibles contratiempos propios de la incorporación de las novedades y en la etapa COVID por las dificultades derivadas de la realización de las sesiones on-line.

Tras esta experiencia (Pedrosa, 2020), las propuestas de mejora se han encaminado a disminuir el número de actos de evaluación y orientar mejor los materiales empleados en la evaluación hacia la medida del nivel de adquisición de la CT13.

Respecto al número de ejercicios evaluables, se ha optado por hacer un único ejercicio individual en una sesión final dedicada exclusivamente a la evaluación. La evolución en este sentido se ha traducido en una cierta mejora, por un lado, se han eliminado aquellas evaluaciones que no llegaron a ser representativas por la falta de entrenamiento de los estudiantes y, por otro lado, los estudiantes dispusieron de más tiempo de trabajo autónomo para profundizar en el manejo del programa antes de realizar la prueba evaluable.

Respecto a los materiales empleados en la evaluación, se modificaron los cuestionarios y se elaboró una rúbrica para la corrección. Ambas acciones se han enfocado a medir mejor la adquisición de la CT13. En los cuestionarios, por ejemplo, se pasó de una única pregunta muy abierta a varias más concretas sobre distintos aspectos clave en el manejo del programa, de esta manera los alumnos pudieron matizar mejor sus respuestas. En la Fig. 1 puede verse un ejemplo concreto sobre el desglose de una pregunta. 
Experiencias y resultados en el desarrollo y validación de instrumentos de evaluación enfocados a la adquisición de la competencia transversal 'CT13 Instrumental Especifica' en asignaturas de Ingeniería Mecánica

Curso 19-20

Justificar cómo se ha realizado el modelo, tanto la definición geométrica como las condiciones de contorno elegidas así como la generación de la malla.

\section{Curso 20-21}

Describe y justifica cómo se ha realizado el modelo en ANSYS:

\begin{tabular}{|l|l|}
\hline $\begin{array}{l}\text { Simplificaciones } \\
\text { en la geometría }\end{array}$ & \\
\hline $\begin{array}{l}\text { Condiciones de } \\
\text { contorno }\end{array}$ & \\
\hline
\end{tabular}

Justifica aquí el criterio de asignación del tamaño de elemento:

Fig. 1 Ejemplo de evolución de una pregunta de un ejercicio evaluable

La Fig. 2 muestra una rúbrica implementada para medir de forma objetiva la bondad de las estrategias seguidas ya que éstas están relacionadas directamente con el nivel de dominio del programa.

\begin{tabular}{|c|c|c|c|c|c|}
\hline CRITERIO & 0-No cumple & 1-Deficiente & 2-Regular & 3-Bueno & 4- Muy bueno \\
\hline $\begin{array}{l}\text { Aplica simetría en } \\
\text { el modelo } \\
\text { geométrico }\end{array}$ & $\begin{array}{l}\text { Comete errores en la } \\
\text { geometría y la modela } \\
\text { completa }\end{array}$ & $\begin{array}{l}\text { Comete errores en la } \\
\text { geometria y a plica alguna } \\
\text { simetría }\end{array}$ & $\begin{array}{l}\text { Modela la pieza } \\
\text { correctamente pero no } \\
\text { aplica ninguna simetría } \\
\text { (completa) }\end{array}$ & $\begin{array}{l}\text { Modela la pieza } \\
\text { correctamente. Simetría } \\
\text { media pieza }\end{array}$ & $\begin{array}{l}\text { Modela la pieza } \\
\text { correctamente. Simetria } \\
\text { un cuarto o un octavo de } \\
\text { pieza }\end{array}$ \\
\hline $\begin{array}{l}\text { Condiciones de } \\
\text { contorno }\end{array}$ & $\begin{array}{l}\text { No aplica condiciones de } \\
\text { contorno }\end{array}$ & $\begin{array}{l}\text { Empotramientos en lugar } \\
\text { de simetrías } \\
\text { No aplica tensión } \\
\text { Le falta alguna condición } \\
\text { de contorno }\end{array}$ & $\begin{array}{l}\text { No fija un punto } \\
\text { (movimiento de sólido } \\
\text { rigido) } \\
\text { Presión con signo positivo }\end{array}$ & $\begin{array}{l}\text { Condiciones de simetría } \\
\text { correctas en los modelos } \\
\text { de media o } 1 / 4 \text { de pieza } \\
\text { Presión correcta }\end{array}$ & $\begin{array}{l}\text { Condiciones de simetría } \\
\text { correctas en } 1 / 4 \text { o } 1 / 8 \text { de } \\
\text { pieza } \\
\text { Presión correcta }\end{array}$ \\
\hline \multicolumn{6}{|l|}{3 MALLADO } \\
\hline \begin{tabular}{|l} 
CRITERIO \\
\end{tabular} & 0-No cumple & 1-Deficiente & 2-Regular & 3-Bueno & 4- Muy bueno \\
\hline $\begin{array}{l}\text { Mallado correcto } \\
\text { con refinamiento }\end{array}$ & No malla & $\begin{array}{l}\text { Malla con elemento } \\
\text { distinto al propuesto } \\
\text { Malla con el elemento } \\
\text { propuesto, pero no aplica } \\
\text { nivel de refinamiento } 1 \text { ni } \\
\text { refina en el concentrador } \\
\text { de tensiones }\end{array}$ & $\begin{array}{l}\text { Malla con el elemento } \\
\text { propuesto pero no aplica } \\
\text { nivel de refinamiento } 1 \text { o } \\
\text { no refina en el } \\
\text { concentrador de tensiones } \\
\text { (solo aplica uno de los dos } \\
\text { refinamientos) }\end{array}$ & $\begin{array}{l}\text { Malla con el elemento } \\
\text { propuesto. } \\
\text { Aplica nivel de } \\
\text { refinamiento } 1 \text { y refina en } \\
\text { el concentrador de } \\
\text { tensiones siguiendo las } \\
\text { instrucciones }\end{array}$ & $\begin{array}{l}\text { Malla con el elemento } \\
\text { propuesto. } \\
\text { Aplica nivel de } \\
\text { refinamiento } 1 \text { y refina en } \\
\text { el concentrador de } \\
\text { tensiones mejorando la } \\
\text { propuesta del enunciado }\end{array}$ \\
\hline \multicolumn{6}{|l|}{4 RESULTADOS } \\
\hline \begin{tabular}{|l|} 
CRITERIO \\
\end{tabular} & 0-No cumple & 1- Deficiente & 2-Regular & 3-Bueno & 4- Muy bueno \\
\hline Solución nodal & No presenta resultados & $\begin{array}{l}\text { No muestra solución } \\
\text { nodal, presenta otra } \\
\text { distinta (elemento) o } \\
\text { la magnitud seleccionada } \\
\text { no representa la solución } \\
\text { requerida (por ejemplo, } \\
\text { desplazamiento) }\end{array}$ & $\begin{array}{l}\text { La solución nodal marcada } \\
\text { en la tabla no es la que } \\
\text { representa la imagen } \\
\text { Error en las unidades de la } \\
\text { tensión }\end{array}$ & Elije una tensión correcta & $\begin{array}{l}\text { Elije una tensión correcta } \\
\text { y justifica que ha buscado } \\
\text { la que se parece más al } \\
\text { resultado de Kt de la } \\
\text { gráfica }\end{array}$ \\
\hline $\begin{array}{l}\text { Cálculo Kt con } \\
\text { ANSYS }\end{array}$ & $\begin{array}{l}\text { No lo hace porque no } \\
\text { dispone de datos }\end{array}$ & $\begin{array}{l}\text { Dispone de los datos pero } \\
\text { no lo presenta }\end{array}$ & $\begin{array}{l}\text { No aplica la corrección de } \\
\text { diámetros } \\
\text { Error de unidades }\end{array}$ & $\begin{array}{l}\text { Hace el cálculo correcto } \\
\text { pero la tensión empleada } \\
\text { no es la óptima }\end{array}$ & Hace el cálculo correcto \\
\hline $\begin{array}{l}\text { Obtención Kt en la } \\
\text { gráfica }\end{array}$ & No lo hace & $\begin{array}{l}\text { Introduce mal los datos de } \\
\text { entrada }\end{array}$ & $\begin{array}{l}\text { Introduce bien los datos, } \\
\text { pero la lectura es } \\
\text { incorrecta }\end{array}$ & Pequeño error & $\begin{array}{l}\text { Lee correctamente el valor } \\
\text { de Kt }\end{array}$ \\
\hline $\begin{array}{l}\text { Justificación de } \\
\text { resultados }\end{array}$ & No escribe nada & $\begin{array}{l}\text { Errores en tensión de } \\
\text { ANSYS o errores en lectura } \\
\text { de gráfica }\end{array}$ & $\begin{array}{l}\text { Justifica el error de no } \\
\text { corregir la tensión nominal } \\
\text { con errores de } \\
\text { discretización } \\
\text { Error de unidades }\end{array}$ & $\begin{array}{l}\text { Cálculos correctos, } \\
\text { justifica errores de lectura } \\
\text { de gráfica o de } \\
\text { discretización }\end{array}$ & $\begin{array}{l}\text { Deja constancia de haber } \\
\text { hecho el cálculo con } \\
\text { distintas tensiones } \\
\text { Propone hacer un refinado } \\
\text { mejor }\end{array}$ \\
\hline
\end{tabular}

Fig. 2 Rúbrica 


\subsection{Prácticas de laboratorio}

Las asignaturas con prácticas de laboratorio son impartidas en el segundo cuatrimestre. La situación sobrevenida de confinamiento total por la pandemia de COVID en el curso 19-20, impidió realizar las sesiones debido a su naturaleza presencial. Actualmente, se están cursando tales prácticas, de modo que en este trabajo se expondrá la metodología, pero no se dispone aún de resultados para hacer una valoración con resultados de la misma.

La herramienta de evaluación propuesta está siendo testeada en dos prácticas de la asignatura de Ciencia de Materiales, correspondiente al tercer curso del Grado en Ingeniería Química y al segundo curso de Grado en Ingeniería de la Energía. Las prácticas seleccionadas son: "Deformación plástica y recocido contra acritud" y "Selección de materiales CES EduPack" (CESEDUPACK). Estas asignaturas pertenecen al nivel 2 de la CT13, cuyo principal resultado de aprendizaje se expresa como: "Integrar correctamente las herramientas básicas del ámbito profesional de forma autónoma" (UPV). Como se observa en la Tabla 2, este nivel de dominio establece dos indicadores diferentes:

1) Manejo de las herramientas básicas de forma autónoma.

2) Selección y combinación de las herramientas básicas adecuadas para realizar un proyecto y/o resolver un problema complejo.

Así mismo, se pretende evaluar el indicador de nivel 1 «identificar las herramientas básicas y su utilidad» por considerarse que completa a los indicadores de nivel 2.

Tabla 2. Resultados de aprendizaje

\begin{tabular}{|c|c|c|c|c|}
\hline $\begin{array}{l}\text { RESULTADO DE } \\
\text { APRENDIZAJE }\end{array}$ & D. No alcanzado & C. En desarrollo & B. Bien/adecuado & $\begin{array}{l}\text { A. Excelente/ } \\
\text { ejemplar }\end{array}$ \\
\hline $\begin{array}{l}\text { 1. Identifica las } \\
\text { herramientas } \\
\text { básicas y su } \\
\text { utilidad. }\end{array}$ & $\begin{array}{l}\text { No identifica las } \\
\text { herramientas } \\
\text { básicas }\end{array}$ & $\begin{array}{c}\text { Identifica las } \\
\text { herramientas } \\
\text { básicas pero no } \\
\text { reconoce su utilidad }\end{array}$ & $\begin{array}{c}\text { Identifica las } \\
\text { herramientas } \\
\text { básicas y su función } \\
\text { principal }\end{array}$ & $\begin{array}{c}\text { Identifica funciones } \\
\text { adicionales de las } \\
\text { herramientas } \\
\text { básicas }\end{array}$ \\
\hline $\begin{array}{l}\text { 2. Maneja las } \\
\text { herramientas } \\
\text { básicas de forma } \\
\text { autónoma. }\end{array}$ & $\begin{array}{l}\text { No es capaz de } \\
\text { manejar las } \\
\text { herramientas sin } \\
\text { unas instrucciones } \\
\text { detalladas }\end{array}$ & $\begin{array}{l}\text { Maneja las } \\
\text { herramientas } \\
\text { siguiendo unas } \\
\text { indicaciones } \\
\text { detalladas }\end{array}$ & $\begin{array}{c}\text { Maneja las } \\
\text { herramientas de } \\
\text { forma autónoma }\end{array}$ & $\begin{array}{c}\text { Maneja las } \\
\text { herramientas con } \\
\text { soltura, explotando } \\
\text { todas sus } \\
\text { funcionalidades }\end{array}$ \\
\hline $\begin{array}{l}\text { 3. Selecciona y } \\
\text { combina las } \\
\text { herramientas } \\
\text { básicas adecuadas } \\
\text { para realizar un } \\
\text { proyecto } \\
\text { profesional y/o } \\
\text { resolver un } \\
\text { problema complejo. }\end{array}$ & $\begin{array}{l}\text { No identifica las } \\
\text { herramientas } \\
\text { adecuadas para el } \\
\text { desarrollo del } \\
\text { proyecto }\end{array}$ & $\begin{array}{c}\text { Identifica las } \\
\text { herramientas a } \\
\text { emplear pero no las } \\
\text { combina de forma } \\
\text { adecuada para el } \\
\text { desarrollo completo } \\
\text { del proyecto }\end{array}$ & $\begin{array}{c}\text { Combina } \\
\text { adecuadamente las } \\
\text { diferentes } \\
\text { herramientas para } \\
\text { completar el } \\
\text { desarrollo del } \\
\text { proyecto }\end{array}$ & $\begin{array}{l}\text { Vislumbra nuevas } \\
\text { formas de combinar } \\
\text { herramientas para } \\
\text { completar el } \\
\text { proyecto de la } \\
\text { forma más } \\
\text { adecuada posible, } \\
\text { valorando sus pros } \\
\text { y contras }\end{array}$ \\
\hline
\end{tabular}

Fuente: UPV

Cada uno de estos resultados de aprendizaje se divide en cuatro niveles de logros o consecución: "D. No alcanzado", “C. En desarrollo", "B. Bueno/adecuado" y “A. Excelente/ejemplar”. Los intervalos numéricos respectivos elegidos son: $0-2.5,2.6-5.0,5.1-7.5,7.6-10$. Por consiguiente, dependiendo de las habilidades 
reflejadas en la calificación total obtenida del cuestionario, el estudiante alcanzará un nivel de logro en la competencia evaluada.

\subsubsection{Metodología}

A partir de la Tabla 2 (de carácter genérico), se ha generado una lista de control. El indicador 2 se evalúa conjuntamente con una pregunta específica en forma de cuestionario tipo lista de verificación y la nota de prácticas. Los indicadores 1 y 3 lo hacen únicamente a partir de preguntas de los cuestionarios tipo lista de verificación. Ambos cuestionarios deben responderse individualmente. Se ha decidido no incluir estos cuestionarios como parte de la puntuación y de forma anónima para detectar en un contexto relajado los conocimientos adquiridos por los estudiantes. Los cuestionarios están basados en trabajos previos de los profesores (Giner-Navarro, 2019; Martinez-Casas, 2019).

El primer cuestionario recoge cuestiones relacionadas con la sesión de prácticas "Deformación plástica y recocido contra acritud" y se realizará al finalizar la práctica. El segundo recoge cuestiones relacionadas con la sesión en aula informática correspondiente a la práctica "Selección de materiales CES EduPack" (CESEDUCPACK) y se realizará en paralelo al desarrollo de la misma.

Las preguntas del cuestionario están destinadas a detectar el grado de comprensión de los estudiantes sobre la utilidad y finalidad del uso de los equipos/programas informáticos empleados en cada una de las sesiones prácticas. Así mismo, se pretende evaluar la destreza de los alumnos para relacionar el uso de dichos equipos y herramientas para ser utilizados como parte de un conjunto con un fin determinado.

Las preguntas están diseñadas bien hacia la selección de equipos que permitan medir una propiedad determinada de un material o bien hacia la selección de secuencias de tratamientos que permitan obtener una propiedad deseada. Este enfoque permitirá dar a conocer el nivel en el que los alumnos han interiorizado las herramientas de las que disponen para cuantificar o modificar las propiedades de los materiales, así como seleccionar los más adecuados para un determinado fin según lo visto en las prácticas. Por tanto, ambos cuestionarios permiten evaluar:

1) La identificación de herramientas básicas y su utilidad.

2) El manejo de las herramientas básicas de forma autónoma.

3) La selección y combinación de las herramientas básicas adecuadas para realizar un proyecto profesional y/o resolver un problema complejo.

Se pretende, por tanto, evaluar los conocimientos previos de los estudiantes y los conocimientos nuevos adquiridos a través de la aplicación práctica del uso de las herramientas vistas en las prácticas de laboratorio seleccionadas.

\section{Resultados}

La incorporación de las metodologías descritas ha permitido hacer una evaluación diferenciada de las prácticas de la asignatura a la de la competencia CT13. Se muestran a continuación las distintas calificaciones en las asignaturas TM y CMS que tienen asignada la evaluación de dicha CT en su plan de estudios. La valoración cualitativa se obtiene a partir de la evaluación numérica obtenida principalmente con las cuestiones relativas al uso del programa contenidas en los cuadernillos de prácticas. En este caso, la escala numérica se ha traducido a los distintos niveles de adquisición con rangos algo distintos a los descritos en las prácticas de laboratorio (apartado 3.2): D (no alcanzado): 0-2.99, C (en desarrollo): 3-5.99, B (adecuado): 6-8.99 y A (excelente): 9-10. La comparativa se muestra en la Fig. 3. 


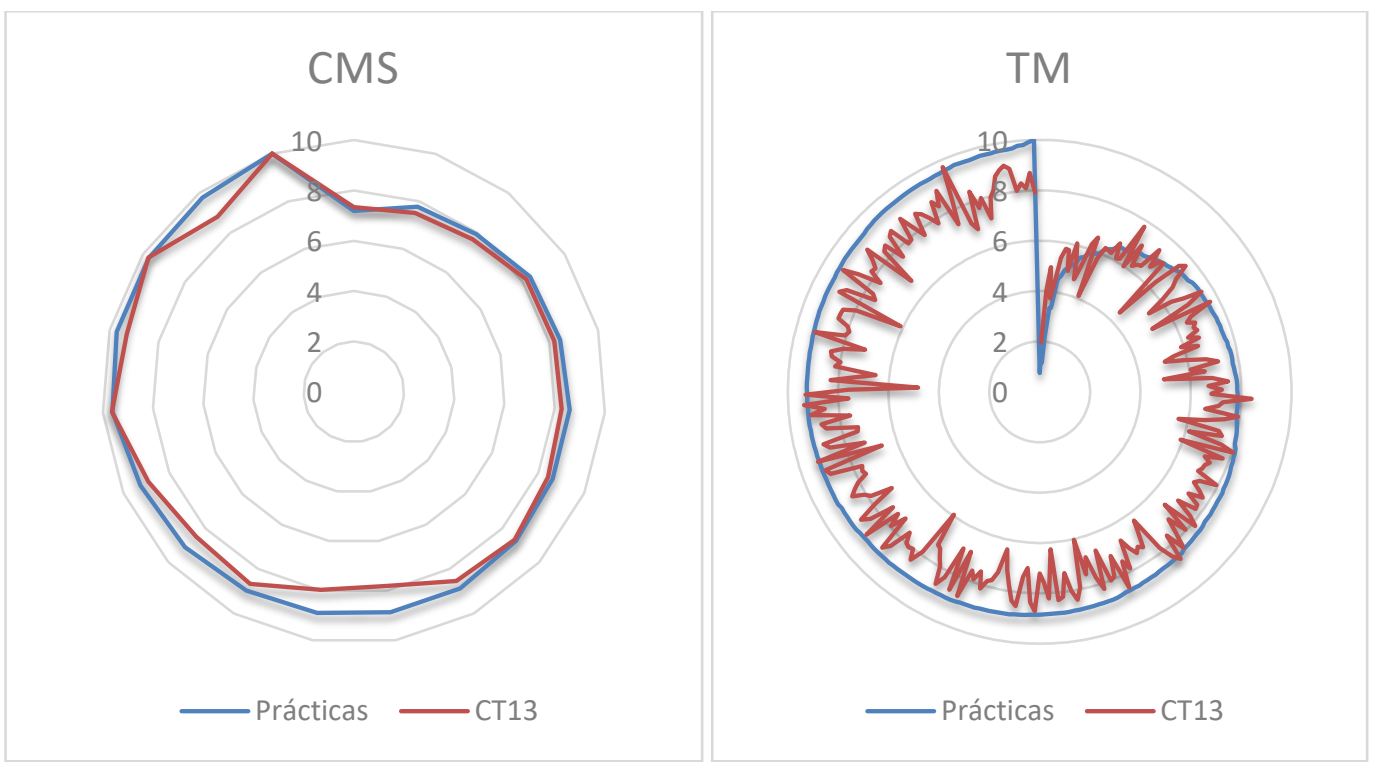

Fig. 3 Comparativa de notas de prácticas y CT13

En ambos casos, la evaluación de las prácticas es superior a la de la CT13, lo que demuestra, por un lado, la importancia de emplear un sistema de evaluación que diferencie el uso correcto de la herramienta y el nivel de dominio de la misma. Una posible explicación a la tendencia observada es que, en general, los alumnos enfocan su esfuerzo más hacia la obtención de los resultados finales que no hacía comprender el proceso.

Por otro lado, al incorporar nuevas cuestiones en la hoja de respuestas que incluyen la justificación de los pasos seguidos, su respuesta no sólo se ha empleado para la medida del grado de adquisición de la CT13, sino que también ha sido tenida en cuenta en la puntuación de la práctica. Se ha hecho una comparativa entre la nota global de la asignatura y la nota de las prácticas. En la Fig. 4 se muestra la comparativa de las medias por curso en la asignatura TM al considerarse más representativa por el número de alumnos matriculados en la misma.

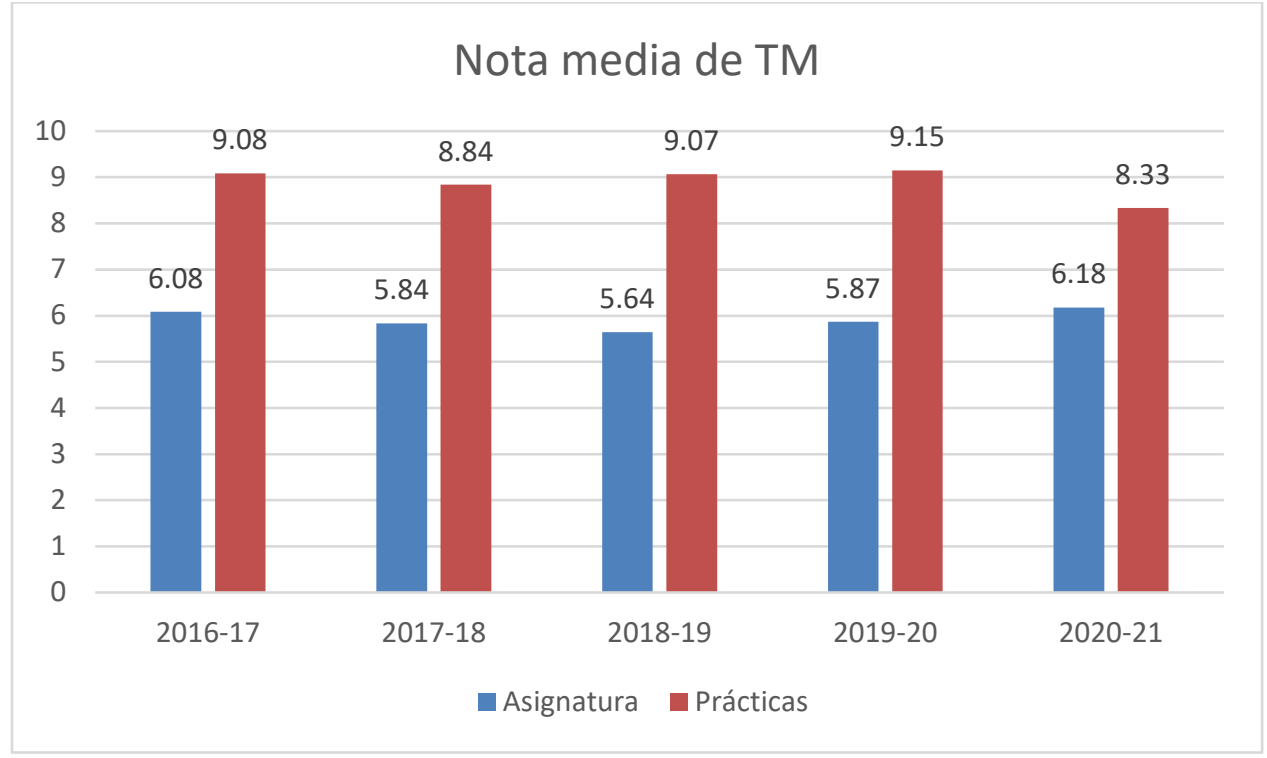

Fig. 4 Comparativa de notas de la asignatura y de las prácticas

(cc) EY-NC-ND 2021, Universitat Politècnica de València

Congreso In-Red (2021) 
En el curso en el que se ha implementado la nueva metodología (20-21) con un ejercicio de resolución individual, no guiado y que los estudiantes realizaron sin la ayuda del profesor, además de registrar una nota media de prácticas inferior, se observa una menor diferencia entre la nota media global de la asignatura y la de prácticas. Dichos resultados parecen razonables, por un lado, al haber aumentado el nivel de exigencia parece lógico que no todos los alumnos puedan alcanzar el nivel excelente, además, las calificaciones obtenidas tienen una distribución normal más parecida a la distribución normal que se suele obtener en la calificación global de la asignatura. Aunque en la gráfica solo se presenta la nota media, la tendencia se correlaciona bastante bien con las notas individuales por estudiante.

\section{Conclusiones}

En este trabajo se describen las experiencias llevadas a cabo en diversas asignaturas del ámbito de la Ingeniería Mecánica. En ellas, se han implementado actividades similares en las sesiones de prácticas con el objetivo de evaluar la competencia transversal Instrumental Específica (CT13), que mide el grado de adquisición de la destreza adquirida por los alumnos en instrumentos de trabajo empleados en el desarrollo profesional de su titulación. El trabajo se ha realizado en dos cursos, permitiendo implementar las novedades en una primera etapa en asignaturas con menos alumnos y, tras realizar un análisis de los resultados, mejorar los aspectos menos satisfactorios e implementarlas en asignaturas con grupos más numerosos. El instrumento escogido en las asignaturas del ámbito de diseño de máquinas es el programa comercial ANSYS, que permite realizar cálculos basados en elementos finitos de problemas de los que se conoce su ecuación de comportamiento, como ocurre en problemas mecánicos. La estrategia empleada ha sido la incorporación de un ejercicio no guiado en la sesión final de prácticas que hasta la fecha seguían un modelo reproductivo exclusivamente. Dicho ejercicio está adaptado al nivel y tipo de problema resuelto en cada asignatura y sesión de prácticas. La idea inicial era que los alumnos, de manera individual, fueran capaces de realizar el trabajo durante cada sesión de prácticas. Sin embargo, la experiencia en el primer curso de implementación demostró que quizás era demasiado precipitado pedir que el alumno manejara el programa con autonomía en la primera sesión, teniendo en cuenta además que, para la mayoría de los alumnos, era la primera vez que manejaban un programa de cálculo de elementos finitos. Por esta razón, en el segundo curso se realizó un único ejercicio en una sesión final de prácticas con el fin de rebajar tanto la presión sobre el alumno como la carga de trabajo de corrección. La experiencia del primer curso también permitió detectar deficiencias en los cuestionarios que han sido mejorados en el segundo curso.

La comparativa entre los resultados de la evaluación del grado de adquisición de la CT13 y las notas prácticas, pone de manifiesto que la nota de prácticas, en algunos casos, no es un buen indicador del grado de adquisición de la competencia, por tanto, es necesario realizar una evaluación diferente. El origen de las discrepancias es diverso, para empezar, el aspecto evaluado es de distinta naturaleza, mientras en las prácticas se busca un resultado numérico correcto de acuerdo con la teoría de la asignatura, en la CT13, se tiene en cuenta la adecuación y la calidad de las estrategias seguidas en la consecución de dicho resultado. Por otro lado, la evaluación de las prácticas es continua y, en ella, contribuye la nota de cada sesión además de la del ejercicio individual, mientras que en la CT13 sólo se tiene en cuenta el ejercicio final evaluable. Finalmente, las sesiones de prácticas están enfocadas al aprendizaje por lo que se consiente un ambiente colaborativo entre alumnos y el profesor aporta a los estudiantes toda la ayuda solicitada durante el desarrollo de las prácticas, mientras que en el ejercicio final es individual y el profesor no responde preguntas sobre los contenidos clave. 


\section{Agradecimientos}

Los autores agradecen la financiación recibida del Vicerrectorado de Estudios, Calidad y Acreditación, el Vicerrectorado de Recursos Digitales y Documentación (proyecto PIME B/19-20/165) y el Instituto de Ciencias de la Educación de la Universitat Politècnica de València (EICE INTEGRAL).

\section{Referencias}

ABET. Accreditation Criteria \& Supporting Documents. $<$ https://www.abet.org/accreditation/accreditation-criteria/> [Consulta: marzo de 2021].

ANDREWS, J. y HIGSON, H. (2008) "Graduate employability, 'Soft skills' versus 'Hard' business knowledge: A European study" en Higher Education in Europe, vol. 33, p. 411-422.

ANECA. Programas de evaluación ACREDITA <http://www.aneca.es/Programas-de-evaluacion/Evaluacion-detitulos/ACREDITA> [Consulta: marzo de 2021].

ANSYS <https://www.ansys.com/> [Consulta: marzo de 2021].

BENEITONE, P. et al (2007). Tuning brochure, Tuning Project: Reflexiones y perspectivas de la Educación Superior en América Latina. Informe Final-Proyecto Tuning-América Latina. Bilbao: Universidad de Deusto. $<$ http://tuning.unideusto.org/tuningal, 2007> [Consulta: marzo de 2021].

CESEDUPACK <https://www.grantadesign.com/education/> [Consulta: marzo de 2021].

ENTWISTLE, N.J. y PETERSON, E.R. (2004) "Conceptions of learning and knowledge in higher education: Relationships with study behaviour and influences of learning environments" en International Journal of Educational Research, vol. 41, p. 407-428.

GINER-NAVARRO, J.; SONSECA, A.; CARBALLEIRA, J. y MARTINEZ-CASAS, J. (2019) "Assessment of instrumental skills and capacity to use the techniques and tools in practice within a subject related to mechanical engineering“. En: 13th International Technology, Education and Development Conference (INTED 2019). Valencia. IATED Academy. 1374 - 1383.

KELLY, A. (2001) "The evolution of key skills: towards a tawney paradigm" en Journal of Vocational Education \& Training, vol. 53, issue 1, p. 21-36. doi: 10.1080/13636820100200149

MARTÍNEZ-CASAS, J.; GINER-NAVARRO, J.; SONSECA, A. y CARBALLEIRA, J. (2019) "Evaluación de las habilidades instrumentales y la capacidad para utilizar las técnicas y herramientas prácticas en el contexto de la ingeniería mecánica". En: XXVII congreso nacional de innovación+docencia+técnica (CUIEET 2019). Alcoy. Universitat Politècnica de València. ISBN 978-84-09-02970-9

MURIAS, P., DE MIGUEL, J.C. y RODRIGUEZ, D. (2007) “A composite indicator for university quality assesment: The case of Spanish higher education system" en Social Indicators research, vol. 89, p. 129-146.

NADAL, E.; RUPEREZ, M.J.; GINER-NAVARRO, J.; ROVIRA, A.; RODENAS, J.J.; MARTINEZ-CASAS, J. y PEDROSA, A.M. (2020). "Assessment of the use of technical software by the students in the context of mechanical engineering". En: 13th International Conference of Education, Research and Innovation (ICERI 2020). Online: IATED Academy. 3344 - 3348.

PEDROSA, A.M.; SANCHEZ-ORGAZ, E.M.; LOZANO-MINGUEZ, E.; MARTINEZ-SANCHIS, S. (2020) "Implementation of activities for the evaluation of the specific instrumental transversal competence in subjects in the area of mechanical engineering". En : 13th International Conference of Education, Research and Innovation (ICERI 2020). Online: IATED Academy. 597-602.

RIECKMANN, M (2012). "Future-oriented higher education: Which key competencies should be fostered through university teaching and learning?" en Futures, vol. 44, p. 127-135.

SURSOCK, A. y SMIDT, H. (2010). Trends 2010: A decade of change in European higher education. Brussels: European University Association.

(cc) EY-NC-ND 2021, Universitat Politècnica de València

Congreso In-Red (2021) 
Experiencias y resultados en el desarrollo y validación de instrumentos de evaluación enfocados a la adquisición de la competencia transversal 'CT13 Instrumental Especifica' en asignaturas de Ingeniería Mecánica

TUNING PROJECT. Tunning General Brochure. <http://www.unideusto.org/tuningeu/documents.html> [Consulta: marzo de 2021].

UPV. Competencias transversales. <http://www.upv.es/contenidos/COMPTRAN/> [Consulta: marzo de 2021].

YOUNG, J. y CHAPMAN, E. (2010) "Generic competency frameworks: A brief historical overview" en Education Research and Perspectives, vol. 37, issue 1, p. 1-24.

ZIENKIEWICZ, O.C. y TAYLOR, R. L. (2006). The Finite Element Method for Solid and Structural Mechanics. 6th ed. Oxford : Elsevier/Butterworth-Heinemann. 